\title{
Philonsorbonne
}

8 | 2014

Année 2013-2014

\section{Espace public et droit administratif}

Francesca DI LASCIO

\section{OpenEdition}

Journals

Édition électronique

URL : https://journals.openedition.org/philonsorbonne/584

DOI : 10.4000/philonsorbonne.584

ISSN : 2270-7336

\section{Éditeur}

Publications de la Sorbonne

\section{Édition imprimée}

Date de publication : 1 janvier 2014

Pagination : 133-143

ISSN : 1255-183X

\section{Référence électronique}

Francesca DI LASCIO, «Espace public et droit administratif », Philonsorbonne [En ligne], 8 | 2014, mis en ligne le 19 janvier 2014, consulté le 08 juin 2021. URL : http://journals.openedition.org/ philonsorbonne/584 ; DOI : https://doi.org/10.4000/philonsorbonne.584 


\title{
Espace public et droit administratif
}

\author{
Francesca DI LASCIO
}

\section{Le contexte}

La notion d' «espace public » n'a pas été, jusqu'à présent, objet d'études spécifiques en droit public, en particulier en droit administratif. De même, il n’y a pas de références fréquentes dans la législation et la jurisprudence.

Récemment, dans le système français, le débat a surgi autour de l'utilisation de cette expression dans la loi n ${ }^{\circ}$ 2010-1192 du 11 octobre 2010. Cette norme a déclaré que dans l'espace public «nul ne peut porter une tenue destinée à dissimuler son visage » (art. 1), indiquant ainsi une limite à l'utilisation des vêtements religieux qui couvrent le visage. En particulier, selon la même loi ; «l'espace public est constitué de voies publiques ainsi que de lieux ouverts au public ou affectés à un service public» (art.2, alinéa 1) : la définition a été critiquée pour son imprécision terminologique et parce qu'elle était susceptible de poser des problèmes considérables dans l'application pratique de l'interdiction ${ }^{1}$. La discussion a eu lieu, cependant, dans une réflexion plus large relative à la relation entre le concept d'espace public et l'exercice de la liberté de religion en Europe pour ce qui concerne l'utilisation de la burqa et d'autres vêtements typiques qui cachent le visage et le corps féminin ${ }^{2}$.

1. Voir O. Bui-Xuan, «L'espace public: l'émergence d'une nouvelle catégorie juridique? Réflexion sur la loi interdisant la dissimulation du visage dans l'espace public », Revue française de droit administratif, 2011, p. 511 et suivantes, qui prend comme exemple celui d'une femme voilée dans sa voiture garée dans une rue très fréquentée ou en transit sur une rue privée à côté d'une rue ouverte au transport public. Voir aussi, du même auteur, Droit et espace(s) public(s), Clermont-Ferrand, Fondation Varenne, 2013.

2. À ce propos voir G. van der Schyff \& A. Overbeeke, «Exercising Religious Freedom in the Public Space: A Comparative and European Convention Analysis of General Burqa Bans », European Constitutional Law Review, 7, 2011, p. 424 et suivantes. 
La notion d'espace public est objet d'attention en droit urbanistique, avec une acception particulière de la notion, à partir de l'œuvre célèbre de Habermas ${ }^{3}$. Dans ce domaine on fait référence à l' «espace public» comme à un espace réel et tangible, partie importante de la sphère publique et espace relationnel effectif, bien qu'immatériel, essentiel pour la construction du système juridique et celle des relations entre les individus de cet espace ${ }^{4}$.

Dans les analyses menées par les différentes sciences sociales sur la notion d' «espace public », on peut donc trouver deux visions de fond : l'une relative au lien avec l'espace physique et l'autre qui est l'expression d'un contenu sociologique et politique ${ }^{5}$. La première des deux acceptions indique, en particulier, les lieux physiques où se développent les échanges sociaux, où se déroule la circulation des personnes, des biens et des capitaux et où les mêmes individus communiquent entre eux. La seconde se réfère à des lieux physiques et symboliques dans lesquels se développent le débat public et la comparaison entre les idées qui contribuent à nourrir la démocratie ${ }^{6}$.

\section{Quelle relation entre la notion d'espace publique et le droit administratif ?}

L'espace public au sens physique est susceptible d'être l'objet d'une nouvelle réflexion dans le domaine juridique et, particulièrement, en droit administratif.

Un premier élément fondamental, auquel il faut prêter attention, est la distinction qui existe dans la structuration des villes entre les espaces publics et les espace privés. Ici se pose le problème de réglementer l'accès à l'espace physique qui se réfère à la notion considérée et il devient fondamental d'établir des critères pour déterminer les droits des individus à être admis dans cet espace. Il en découle une relation juridique entre l'espace physique (le sol) et le droit, traditionnellement basée sur le concept de la propriété individuelle, qui est «le moyen essentiel par lequel le Droit rattache des droits et des obligations à des choses et des lieux : en indiquant qui possède quel type de droits, et d'obligations, à un endroit donné ${ }^{7} \gg$.

\footnotetext{
3. J. Habermas, L'espace public. Archéologie de la publicité comme dimension constitutive de la société bourgeoise, Paris, Payot, [1962] 1998.

4. Pour les principales définitions de l'«espace public» données par les urbanistes, voir C. Iaione, «La città come bene comune », Aedon, 1, 2013.

5. Voir J.-B. Auby, «Espace public, espaces publics», Droit administratif, Juillet 2009, repère p. 3.

6. L'affirmation d'un lien entre la polysémie de l'expression et la distinction entre espace public et espaces publics a été signalée par T. Paquot, L'espace public, Paris, La Découverte, 2009.
}

7. Voir encore J.-B. Auby, op. cit., p. 21. 
Si la propriété peut être le critère utilisé pour différencier l'espace public de l'espace privé, il suffit de vérifier l'appartenance d'un certain espace ou d'un groupe de biens à un organisme public ou, à l'inverse, privé pour qualifier un espace donné et définir le cadre juridique ici applicable $^{8}$. Mais la relation entre les institutions publiques et les espaces physiques est établie aussi par la nécessité d'identifier des règles et des outils destinés à rendre plus efficace la gestion des espaces et des biens publics qui y sont présents. En effet, la gestion des biens selon des critères visant à en améliorer l'utilisation devient urgente à cause de la crise économique et financière, qui exige un gros effort des États pour garantir la stabilité des finances publiques et pour soutenir la reprise du développement économique.

Le lien étroit entre la finance et les biens publics, ainsi que la relation entre eux, prennent de l'importance ${ }^{9}$. La connexion avec la stabilité des finances publiques est notable parce que certains biens contribuent à la composition du patrimoine de l'État et des collectivités territoriales. D'autres biens, cependant, peuvent potentiellement générer des revenus importants puisque leur utilisation est soumise à des frais (concessions de plage) ou à des péages (autoroutes). L'influence des biens publics sur la reprise du développement économique est importante, surtout de ceux qui appartiennent au domaine public, puisqu'ils peuvent être accordés pour l'utilisation à des fins de développement d'activités commerciales et industrielles (concessions pour l'exploitation des sources d'eau minérale et thermale).

À la valorisation optimale des biens doit, cependant, toujours correspondre la protection de leur intégrité et de leurs caractéristiques typiques, pour en garantir la protection effective contre l'utilisation massive. Considérons, par exemple, une partie de la plage objet de concession pour l'installation d'une industrie; un exemple qui montre l'importance des questions liées à la préservation de l'environnement, lesquelles ont valeur en dehors des frontières nationales ${ }^{10}$.

Comme second critère d'analyse, on doit considérer qu'il y a une distinction, indépendante du type de propriété, entre les situations juridiques subjectives qui se présentent dans les lieux publics, ceux ouverts au public et ceux exposés au public. Les catégories visées, en fait, font l'objet de règles juridiques susceptibles de s'exercer aussi dans les espaces qui sont de propriété privée. L'hypothèse exposée émerge avec une plus grande clarté

\section{Ibid., p. 21.}

9. Comme dit G. Della Cananea, «I beni », in S. Cassese (éd.), Istituzioni di diritto amministrativo (4 édition), Milan, Giuffrè Editore, 2012, p. 225.

10. Comme le démontrent les politiques européennes pour la gestion intégrée des zones côtières, le changement climatique et l'utilisation des énergies durables. En ce domaine l'importance du rôle des collectivités territoriales va devenir évidente. Voir N. Rangone \& J. Ziller (éd.), Politiche e regolazioni per lo sviluppo locale sostenibile. Il patto dei sindaci, Naples, Editoriale Scientifica, 2013. 
si nous rapportons le contenu des trois catégories mentionnées, comme conçu pour la présente analyse : un lieu public est un espace destiné à un usage général et auquel les individus accèdent librement (par exemple, la plage); un lieu ouvert au public est un espace dont l'accès est limité et soumis à des règles spécifiques établies par le propriétaire, des lois ou des actes administratifs sectoriels (par exemple, les horaires d'ouverture et le paiement du billet d'entrée à un musée) et un lieu exposé au public est un espace soumis à la vision, comme le sont les biens qui y sont contenus et les événements qui y arrivent (par exemple, une place ou un centre commercial). Cette classification montre que la nature de la propriété des biens n'est pas le seul critère de régulation administrative mais que le droit peut imposer des obligations et des limites «transversales », car étroitement liées à la fonctionnalité des biens.

Cela est particulièrement évident pour certaines tendances dans l'utilisation d'espaces publics ouverts au public, en liaison avec de nouveaux phénomènes privés (nouvelles façons de se déplacer, nouveaux modes de divertissement, nouvelles formes de spectacles et de rencontres) ${ }^{11}$. On peut également penser à la nécessité de formes de régulation originales pour les problèmes liés aux questions de sécurité publique (surveillance vidéo des lieux) ou les problèmes hygiéniques et sanitaires, ou encore à l'interdiction de fumer, dont l'application dans les lieux ouverts et dans ceux exposés au public est justifiée par la nécessité de protéger la santé de ceux qui y passent. En ce sens, on détecte l'obligation d'exercer une fonction administrative de protection d'espaces publics qui est indépendante de leur classement et du statut de la propriété publique ou privée ${ }^{12}$. À cette fin, l'administration compétente dans la régulation de l'espace physique peut imposer des limites, faire usage de pouvoirs autoritaires pour contrôler et, si nécessaire, exercer des pouvoirs de police et de sanction administrative.

Une dernière remarque concerne non la protection qui doit être accordée à la conservation optimale de l'espace et des biens qui s'y trouvent, mais les choix concernant l'utilisation des biens par la communauté. Cette dernière, en effet, est naturellement porteuse d'un intérêt qui se réalise, dans l'abstrait, à travers une utilisation générale (c'est-à-dire sans aucune restriction) et gratuite. Ces deux fonctions peuvent, cependant, ne pas exister ou ne subsister qu'en partie car l'identification de la meilleure utilisation à faire des biens de sa propriété est laissée à la discrétion du propriétaire. Par rapport à la prise en charge de ce choix, cependant, il est maintenant essentiel que soient utilisés des mécanismes de décisions qui ménagent

11. Voir J.-B. Auby, op. cit., p. 43.

12. Un autre exemple, concernant des bénéficiaires plus limités, est donné par les contraintes qui imposent la modification des espaces publics consécutive aux règles relatives à l'accessibilité des personnes handicapées. Ces règles peuvent exiger, en effet, la mise en place de structures spécifiques et l'adaptation à certaines mesures et dimensions spécifiques. Voir F. Rolin, «L'espace public en droit administratif », in O. Bui-Xuan, op. cit., p. 62-63. 
une place à la société civile et, en particulier, à la société locale concernée par le rapport avec l'espace physique où se trouve le bien intéressé.

Après avoir décrit le cadre de référence, il est possible d'étudier plus en détail les domaines du droit administratif qui semblent le plus se prêter à décrire la relation entre ce cadre juridique et la notion d'espace public.

\section{L'espace public comme ensemble de biens domaniaux}

Dans une première hypothèse, l'espace public peut être considéré comme un lieu physique dans lequel il y a les «res en ordre auxquelles un sujet peut avoir un intérêt important et sur lequel il est possible de construire une relation juridique » et dont l'ensemble est défini par le terme «biens ${ }^{13}$. Il y a donc une distinction entre la chose (le bien) et les valeurs qui peuvent découler de celle-ci, l'imputation desquelles, comme mentionné, est liée à la relation juridique ci-dessous. Dans ce contexte, les biens peuvent être distingués grâce à deux critères : quels sujets ont sur eux des droits réels (dont le plus important : le droit de propriété), ou en raison du cadre juridique.

Malgré cela, il subsiste un régime de spécialité pour les biens qui concernent les intérêts de la communauté, à partir de laquelle, comme nous l'avons mentionné précédemment, découle la mise en place de restrictions à la disposition et à l'utilisation du même bien, comme à l'accès à la ressource. On peut donc dire que le caractère le plus important des biens publics consiste dans leur capacité à fournir des utilités juridiquement considérables en relation à un ou plusieurs intérêts publics.

La relation entre les biens et les intérêts publics se caractérise principalement de deux façons : pour la première, l'intérêt relatif au bien est instrumental par rapport à son aptitude à servir à l'exercice d'une fonction ou d'un service public. Ici, les biens sont considérés comme un moyen pour l'administration (la propriété militaire, les véhicules de transport public, etc.) ; pour la seconde, le bien réalise immédiatement un intérêt collectif (les musées, les rues et le rivage de la mer) ${ }^{14}$. Si cela arrive, il est possible de justifier la limitation de certaines facultés typiques des droits dominicaux qui reviennent aux personnes privées en relation avec les biens (les contraintes imposées au jus aedificandi sur le sol public) ${ }^{15}$.

En droit italien, la principale source de réglementation des biens publics se trouve dans le Code civil adopté en 1942, qui les classe en fonction

13. Voir G. Rossi, Principi di diritto amministrativo, Turin, Giappichelli, 2010, p. 241.

14. Mais ils peuvent être aussi des biens qui ont les deux caractères. Voir M. Dugato, «Il regime dei beni pubblici: dall'appartenenza al fine », in A. Police (éd.), I beni pubblici : tutela, valorizzazione e gestione, Milan, Giuffrè Editore, 2008, p. 45-46.

15. Voir G. della Cananea, op. cit., p. 227. 
de l'intensité des contraintes mentionnées précédemment et qui soumet la catégorie des biens domaniaux aux dispositions les plus strictes. Ce dernier cas comprend des biens à l'égard desquels les pouvoirs publics peuvent restreindre la circulation ainsi que la transformation, affectant ainsi plus clairement l'espace public. Cette considération concerne, en particulier, les biens domaniaux naturels, qui comprennent les biens déjà existants dans la nature et sont distincts de biens domaniaux artificiels, qui se réfèrent aux biens construits par l'homme et destinés à satisfaire une fonction publique.

Les biens domaniaux sont inaliénables et, par conséquent, les personnes privées ne peuvent pas avoir de droits de propriété sur eux : elles ne peuvent demander que leur usage temporaire. Les mêmes biens sont indisponibles et, par conséquent, ne peuvent pas faire l'objet d'autres droits en faveur de tiers, comme dans le cas de la dette par l'administration. En outre, la personne publique propriétaire détient des pouvoirs étendus de protection : elle peut faire usage des moyens ordinaires de défense de la propriété privée et avoir des facultés de police domaniale à travers lesquelles imposer, par exemple, la suppression des artefacts construits sur le sol public en violation de la loi.

La discipline de l'utilisation et de l'accès aux biens domaniaux présente un intérêt particulier. Comme nous l'avons dit, le système juridique encadre l'utilisation collective de biens et leur utilisation temporaire par les propriétaires privés. Il est possible d'identifier quatre types d'utilisation: l'utilisation directe par le propriétaire public, qui implique des sanctions dans le cas de l'utilisation par d'autres (par exemple, l'accès aux biens militaires ou aux bureaux publics est réservé uniquement aux employés) ; l'utilisation mixte (par exemple, les routes militaires sur lesquelles le passage est public) ; l'utilisation générale lorsque l'usage du bien est permis au profit de la communauté (par exemple, le domaine hydrique ou les biens d'intérêts historiques), librement ou sous condition de la délivrance d'une somme (par exemple, les autoroutes à péage) ou d'une autorisation spécifique (par exemple, le rejet dans les eaux publiques); l'utilisation particulière lorsque le bien est mis à la disposition de propriétaires privés pour une période temporaire avec un acte administratif de concession, dont la durée est déterminée à l'avance à travers une procédure administrative d'évaluation comparative et contre paiement au titulaire d'un droit (les réserves de pêche $)^{16}$. Les différents types d'usages peuvent coexister, comme c'est le cas si l'administration prévoit l'octroi d'une partie du bien sans en entraver l'utilisation générale.

Dans le dernier cas, certains des problèmes déjà exposés se font plus nombreux, car l'utilisation particulière repose sur l'adoption d'une concession qui permet au privé la jouissance exclusive d'une partie des bénéfices résultant du bien public; à l'expiration duquel les biens peuvent être adressés au même type d'utilisation ou à un autre.

16. Voir, à ce propos, F. Di Lascio, «Concessioni di demanio marittimo e tutela della concorrenza », in Forum TAR, 3, 2009, p. 787 et suivantes. 


\section{L'espace public et les biens communs}

Le deuxième aspect à considérer est la relation entre la notion d'espace public et les biens communs, à travers lesquels l'état de bien-être et les droits fondamentaux des citoyens peuvent se réaliser.

L'intérêt pour cette question a commencé à prendre de l'importance en raison de l'émergence, de plus en plus fréquente, au niveau national mais aussi international, du problème relatif à l'utilisation et au contrôle des ressources naturelles, considérées comme biens vitaux pour les communautés locales de référence. Il s'agit de biens librement accessibles et qui donnent lieu à des phénomènes de surconsommation qui, s'ils ne sont pas contrés, pourront conduire à l'épuisement progressif des ressources ${ }^{17}$ : ces biens sont de plus en plus définis par les médias ainsi que par la communauté scientifique et universitaire par le terme de «biens communs $»^{18}$.

Le débat sur cette notion fait l'objet d'une approche interdisciplinaire qui implique son examen par plusieurs méthodes d'enquête, critères et approches. Il s'ensuit que, lorsque l'analyse théorique est appelée à apporter sa contribution à l'identification de solutions pratiques, de nombreuses difficultés se présentent. Si cet aspect est très évident dans la comparaison des théories économiques entre les biens communs et les biens publics juridiques, la première catégorie étant référée à un ensemble beaucoup plus large et varié que la deuxième, les mêmes conflits se retrouvent dans l'analyse juridique, dans laquelle émergent parfois des contradictions. Par exemple, on constate que la relation entre biens publics et biens communs a des connotations différentes selon qu'elles sont examinées selon la perspective du droit public ou privé.

Au cours des dernières années, le sujet a été discuté en Italie, dans ses aspects juridiques, notamment dans le débat sur la propriété publique menée

17. Il s'agit du modèle économique nommé «tragedy of commons », décrit pour la première fois par G. Hardin, « The Tragedy of the Commons », Science, ${ }^{\circ}$ 162, 1968, p. 1243 et suiv., pour lequel il est nécessaire de limiter le libre accès et l'exploitation sans critère des biens naturels si on veut éviter leur disparition. En droit italien, voir G. Napolitano, «I beni pubblici e le "tragedie dell'interesse comune" ", in AIPDA, Annuario 2006. Analisi economica e diritto amministrativo, Milano, Giuffrè Editore, 2007, p. 125 et suivantes.

18. La littérature sur le sujet est vaste. Parmi la littérature non-juridique, on doit rappeler E. Ostrom, Governare i beni collettivi, Venise, Marsilio Editore, 2006, ainsi que E. Ostrom \& C. Hess (éd.), Understanding Knowledge as a Commons: From Theory to Practice, Cambridge, MIT press, 2006. Dans la littérature juridique italienne voir A. Lucarelli, La democrazia dei beni comuni, Roma-Bari, Laterza, 2013; U. Mattei, Beni comuni. Un manifesto, Roma-Bari, Laterza, 2012 ; M.R. Marella (éd.), Oltre il pubblico e il privato. Per un diritto dei beni comuni, Vérone, Ombre Corte, 2012; S. Rodotà, I beni pubblici. Dal governo democratico dell'economia alla riforma del codice civile, Rome, Accademia Nazionale dei Lincei, 2010. Voir aussi, pour une approche interdisciplinaire, G. Napolitano \& M. Abrescia, Analisi economica del diritto pubblico, Bologne, Il Mulino, 2009, en particulier les p. 64-67, 71-73 et 86-89. 
par la commission Rodotà ${ }^{19}$. Cette organisation a défini les biens communs comme «les choses qui expriment des utilités fonctionnelles à l'exercice des droits fondamentaux et au libre développement de la personne », sanctionnant la nécessité de leur protection sur le plan juridique, même sur une base prospective, pour le bénéfice des générations futures. Le concept indiqué identifie, par conséquent, les biens communs comme des instruments de la réalisation des droits fondamentaux des personnes appartenant à une communauté donnée.

Dans les lignes directrices énoncées par la commission sur le régime de propriété de ces biens ressort la corrélation forte avec la notion d'espace public. En effet, les biens communs peuvent être la propriété de l'administration ou d'une personne privée : dans le premier cas, elles doivent suivre les règles générales des biens domaniaux ; dans le second, leur valeur ne peut être mesurée en termes seulement économiques: leur utilisation par la communauté doit aussi être prise en considération. Pourtant, quel que soit leur régime de propriété, les biens communs et l'espace public qui les contient doivent être soignés et protégés.

\section{L'espace public comme lieu d'exercice de la fonction de régulation administrative}

Dans un second sens, l'espace public peut être défini comme un lieu physique d'exercice des fonctions administratives. Ou plutôt, l'espace public, comme espace dans lequel se joue l'interaction de nombreux intérêts, dont certains se qualifient comme publics, nécessite que les institutions publiques s'occupent de leur réalisation. Dans ce cas, les fonctions du droit administratif ne sont pas, par conséquent, étroitement liées aux droits de propriété : les fonctions auxquelles il se réfère se rapportent plus directement à la finalisation de biens, en insistant sur l'espace physique considéré. Pour mieux comprendre l'impact qu'un tel concept a sur le réel, on peut se référer à deux exemples.

Dans le droit italien, le paysage est l'objet de plusieurs définitions ; en particulier, le décret législatif $n^{\circ} 42 / 2004$ se réfère au paysage comme ensemble de biens désigné par une loi, par une mesure administrative ou par le plan d'aménagement paysager ${ }^{20}$.

Ce dernier instrument est au centre de l'affaire concernant la zone archéologique de Tuvixeddu-Tuvumannu, qui contient une importante

19. La commission, créée au sein du ministère de la Justice le 21 Juin 2007, avec la tâche de réformer le régime juridique des biens publics et d'adapter les règles du Code civil à l'évolution économique, technologique et réglementaire, a conclu ses travaux en soumettant un projet de loi qui, cependant, n'a pas encore été suivi d'effet.

20. Voir G. Sciullo, «Il paesaggio. Report annuale - Italia - $2011 »$, IUS Publicum Network Review, p. 3. 
nécropole située dans la Région Sardaigne. La question portait sur l'annulation d'une décision du gouvernement régional, requise par la commune de Cagliari à laquelle appartient la zone archéologique, et concernant une certaine étendue spatiale définie comme bien du paysage ; cela a rendu nécessaire l'application de moyens spécifiques de protection (soumission de tous les bâtiments à l'acquisition préalable d'un titre de dégagement archéologique). Or, la commune avait conclu des accords avec des entreprises de construction pour la réalisation d'un centre commercial sur la zone protégée : sa construction est devenue impossible, suite à la nouvelle délimitation archéologique.

Donc, à travers le plan d'aménagement paysager, une protection pour la conservation de certaines fonctionnalités de la valeur des paysages et du patrimoine historique et culturel peut être imposée à une zone spécifique : la fonction de protection que l'administration exerce domine sur les destinations urbanistiques affectées au territoire et implique ainsi la capacité à définir, par voie administrative, l'espace physique. Le droit administratif exerce ainsi un rôle actif dans la détermination de la dimension tangible attribuée à l'espace public.

Dans la relation entre ville et droit, il est également possible d'identifier trois domaines d'analyse : le lien entre le droit et la physicalité de la ville, entre la loi et le dynamisme de la ville et entre le droit et la politique de la ville. C'est dans le premier des trois que l'élément du territoire, à travers lequel les villes sont reconnues comme des objets juridiques particuliers, s'exprime avec force : ici on retrouve l'essence même de la relation entre le droit et la ville car la loi définit des espaces auxquels s'attachent les règles relatives à la propriété, au gouvernement du territoire et aux collectivités territoriales ${ }^{21}$.

En ce même sens, la ville est décrite comme lieu physique d'interaction où surgissent deux types des problèmes : la séparation et l'identification des espaces, qui impliquent la nécessité d'indiquer quelles devraient être les limites entre espaces publics et privés; la détermination des règles pour gérer l'interaction et la coexistence entre les espaces publics et privés (par exemple, en référence à la transition entre l'un et l'autre, à l'impact sur la vue, à leur entretien). C'est dans ce contexte qu'émerge la notion d' « espace public ».

On doit aussi rappeler que la structuration de la ville est toujours une division établie dans les espaces urbains publics et privés qui est variable et inconstante et change d'un système social et juridique à l'autre. Toutefois, dans le cycle actuel de l'histoire, nous assistons, d'une part, à la croissance des espaces publics au détriment des privés; d'autre part, le processus de privatisation des biens publics et l'externalisation de fonctions administratives, motivées par la nécessité de valoriser les biens comme les ressources économiques, impliquent la réduction de l'espace public en

21. Voir encore J.-B. Auby, op. cit., p. 4. 
faveur de l'espace privé. Globalement, donc, la relation fonctionnelle entre ces deux types d'espaces au sein des villes ne cesse de se complexifier ${ }^{22}$.

\section{Conclusions}

À l'issue de cette analyse, il est possible de faire quelques commentaires concernant la relation entre le droit administratif et l'espace public et, en particulier, la contribution que le premier peut apporter à la construction d'une notion juridique du second.

Tout d'abord, les trois cas examinés expriment d'une manière différente la construction de la relation entre l'administration publique et les personnes privées et, par conséquent, la relation, en général, entre le public et le privé. Quand on parle de l'espace public comme d'un ensemble de biens domaniaux, ce rapport est plus traditionnel puisque l'administration dispose de larges pouvoirs discrétionnaires pour décider de la destination des biens et, par conséquent, peut également limiter de manière significative la satisfaction des intérêts individuels à cet égard. Il convient toutefois de garder à l'esprit la nécessité, de plus en plus urgente, que les institutions prennent en compte des options de choix permettant la valorisation optimale de biens, même en termes économiques, sans affecter leur conservation et leur protection.

Dans l'espace public comme lieu d'exercice de la fonction administrative, la relation avec les citoyens est plus équilibrée car les institutions doivent prendre en compte, d'une part, les espaces privés qui existent déjà et, d'autre part, doivent impliquer les citoyens dans les choix sur l'emplacement de nouveaux espaces publics et des biens qui y seront placés. De plus, les citoyens doivent être impliqués dans la gestion active et partagée de ces espaces et aussi des espaces privés susceptibles d'exiger l'exercice de puissance publique.

Enfin, dans l'espace public comme bien commun, émerge clairement la scission entre titre de la propriété du bien et attitude à l'égard de la réalisation des droits fondamentaux, le premier élément étant indifférent à la poursuite de celle-ci.

On est, ainsi, en train d'assister à « un changement profond. Droits fondamentaux, accès, biens communs dessinent une intrigue qui redéfinit les relations entre le monde des humains et le monde des biens ». Ou bien encore: "La propriété, qu'elle soit publique ou privée, ne peut pas comprendre et épuiser la complexité du rapport entre personne et biens. Un ensemble de relations est désormais confié à des logiques non-propriétaires ${ }^{23} \gg$.

22. Voir J.-B. Auby, op. cit., p. 20-25.

23. Voir S. Rodotà, Il diritto di avere diritti, Roma-Bari, Laterza, 2012, p. 109-110. 
L'étude de la notion d'espace public avec les catégories et les critères du droit administratif peut donc servir à renforcer ou accélérer certains changements qui sont déjà en cours, comme ceux relatifs à la valorisation des biens, à leur gestion partagée et participative et à la reconnaissance d'une troisième catégorie dans le régime de la propriété. Mais il s'agit d'un concept peu exploré par la science du droit public ; rien n'empêche que son potentiel soit encore plus grand ou qu'il puisse servir à initier une étude concernant de nouveaux modèles de relation entre l'administration publique et les citoyens. 